\title{
Boundedness for fractional Hardy-type operator on variable-exponent Herz-Morrey spaces
}

\author{
Jiang-Long Wu and Wen-Jiao Zhao
}

\begin{abstract}
In this article, the fractional Hardy-type operator of variable order $\beta(x)$ is shown to be bounded from the variable-exponent Herz-Morrey spaces $M \dot{K}_{p_{1}, q_{1}(\cdot)}^{\alpha(\cdot), \lambda}\left(\mathbb{R}^{n}\right)$ into the weighted space $M \dot{K}_{p_{2}, q_{2}(\cdot)}^{\alpha(\cdot), \lambda}\left(\mathbb{R}^{n}, \omega\right)$, where $\alpha(x) \in L^{\infty}\left(\mathbb{R}^{n}\right)$ is log-Hölder continuous both at the origin and at infinity, $\omega=(1+|x|)^{-\gamma(x)}$ with some $\gamma(x)>0$, and $1 / q_{1}(x)-1 / q_{2}(x)=\beta(x) / n$ when $q_{1}(x)$ is not necessarily constant at infinity.
\end{abstract}

\section{Introduction}

Let $f$ be a locally integrable function on $\mathbb{R}^{n}$. The $n$-dimensional Hardy operator is defined by

$$
\mathscr{H}(f)(x):=\frac{1}{|x|^{n}} \int_{|t|<|x|} f(t) d t, \quad x \in \mathbb{R}^{n} \backslash\{0\} .
$$

In 1995, Christ and Grafakos [3] obtained the result for the boundedness of $\mathscr{H}$ on $L^{p}\left(\mathbb{R}^{n}\right)(1<p<\infty)$ spaces, and they also found the exact operator norms of $\mathscr{H}$ on this space. In 2007, Fu et al. [12] gave the central bounded mean oscillation (BMO) estimates for commutators of $n$-dimensional fractional and Hardy operators. Recently, the first author [32], [29], [31], [35], [33], [34], [30] has also considered the boundedness for the Hardy operator and its commutator in (variable-exponent) Herz-Morrey spaces.

Lately, there has been an increase in the number of investigations related to both the theory of the variable-exponent function spaces and the operator theory in these spaces. This is caused by possible applications to models with nonstandard local growth (in elasticity theory, fluid mechanics, differential equations, and image processing; see, e.g., [24], [11], [2], [13], [25] and references therein) and is based on the breakthrough result on the boundedness of the Hardy-Littlewood

Kyoto Journal of Mathematics, Vol. 56, No. 4 (2016), 831-845 DOI 10.1215/21562261-3664932, (C) 2016 by Kyoto University

Received May 11, 2015. Revised November 5, 2015. Accepted November 6, 2015. 2010 Mathematics Subject Classification: Primary 42B20; Secondary 47B38.

Wu's work was supported in part by the National Natural Science Foundation of China (Grant No. 11571160) and Mudanjiang Normal University (No. QY2014007). Zhao's work was supported in part by Mudanjiang Normal University (No. QY2014007). 
maximal operator in these spaces (for more details see [19], [4]-[6], [9], [7], [10], [18], [20], [22], [23], and others).

We first define the $n$-dimensional fractional Hardy-type operators with variable order $\beta(x)$ as follows.

\section{DEFINITION 1.1}

Let $f$ be a locally integrable function on $\mathbb{R}^{n}, 0 \leq \beta(x)<n$. The $n$-dimensional fractional Hardy-type operators of variable order $\beta(x)$ are defined by

$$
\begin{aligned}
& \mathscr{H}_{\beta(\cdot)}(f)(x):=\frac{1}{|x|^{n-\beta(x)}} \int_{|t|<|x|} f(t) d t, \\
& \mathscr{H}_{\beta(\cdot)}^{*}(f)(x):=\int_{|t| \geq|x|} \frac{f(t)}{|t|^{n-\beta(x)}} d t,
\end{aligned}
$$

where $x \in \mathbb{R}^{n} \backslash\{0\}$.

Obviously, when $\beta(x)=0, \mathscr{H}_{\beta(\cdot)}$ is just $\mathscr{H}$, and we denote by $\mathscr{H}^{*}:=\mathscr{H}_{\beta(\cdot)}^{*}=$ $\mathscr{H}_{0}^{*}$. When $\beta(x)$ is constant, $\mathscr{H}_{\beta(\cdot)}$ and $\mathscr{H}_{\beta(\cdot)}^{*}$ will become $\mathscr{H}_{\beta}$ and $\mathscr{H}_{\beta}^{*}$, respectively (see [12]).

The Riesz-type potential operator with variable order $\beta(x)$ is defined by

$$
I_{\beta(\cdot)}(f)(x)=\int_{\mathbb{R}^{n}} \frac{f(y)}{|x-y|^{n-\beta(x)}} d y, \quad 0<\beta(x)<n .
$$

In 2004, Diening [8] proved Sobolev's theorem for the potential $I_{\beta}$ on the whole space $\mathbb{R}^{n}$ assuming that $p(x)$ is constant at infinity $(p(x)$ is always constant outside some large ball) and satisfies the same logarithmic condition as in [26]. Another progress for unbounded domains is the result of Cruz-Uribe et al. [6] on the boundedness of the maximal operator in unbounded domains for exponents $p(x)$ satisfying the logarithmic smoothness condition both locally and at infinity.

Kokilashvili and Samko [17] proved a Sobolev-type theorem for the potential $I_{\beta(\cdot)}$ from the space $L^{p(\cdot)}\left(\mathbb{R}^{n}\right)$ into the weighted space $L_{\omega}^{q(\cdot)}\left(\mathbb{R}^{n}\right)$ with the power weight $\omega$ fixed to infinity, under the logarithmic condition for $p(x)$ satisfied locally and at infinity, not supposing that $p(x)$ is constant at infinity but assuming that $p(x)$ takes its minimal value at infinity.

In addition, the theory of function spaces with variable exponents has rapidly made progress in the past 20 years since some elementary properties were established by Kováčik and Rákosník [19].

In 2012, Almeida and Drihem [1] discussed the boundedness of a wide class of sublinear operators on Herz spaces $K_{q(\cdot)}^{\alpha(\cdot), p}\left(\mathbb{R}^{n}\right)$ and $\dot{K}_{q(\cdot)}^{\alpha(\cdot), p}\left(\mathbb{R}^{n}\right)$ with variable exponents $\alpha(\cdot)$ and $q(\cdot)$. Meanwhile, they also established Hardy-LittlewoodSobolev theorems for fractional integrals on variable Herz spaces. In 2013, Samko [28], [27] introduced a new Herz-type function space with variable exponent, where all three parameters are variable, and proved the boundedness of some sublinear operators (see also [15]). In 2014, Izuki and Noi [16] examined the duality and reflexivity of Herz spaces $K_{q(\cdot)}^{\alpha(\cdot), p(\cdot)}\left(\mathbb{R}^{n}\right)$ and $\dot{K}_{q(\cdot)}^{\alpha(\cdot), p(\cdot)}\left(\mathbb{R}^{n}\right)$ with variable 
exponents. Recently, $\mathrm{Wu}[30]$ considered the boundedness for fractional Hardytype operators on Herz-Morrey spaces $M \dot{K}_{p, q(\cdot)}^{\alpha(\cdot), \lambda}\left(\mathbb{R}^{n}\right)$ with variable exponent $q(\cdot)$ but fixed $\alpha \in \mathbb{R}$ and $p \in(0, \infty)$.

Motivated by the above results, we investigate mapping properties of the fractional Hardy-type operators $\mathscr{H}_{\beta(\cdot)}$ and $\mathscr{H}_{\beta(\cdot)}^{*}$ within the framework of the variable-exponent Herz-Morrey spaces $M \dot{K}_{p, q(\cdot)}^{\alpha(\cdot), \lambda}\left(\mathbb{R}^{n}\right)$.

Throughout this article, we will denote by $|S|$ the Lebesgue measure and by $\chi_{S}$ the characteristic function for a measurable set $S \subset \mathbb{R}^{n}$. We let $B(x, r)$ be the ball centered at $x$ and of radius $r$, and we let $B_{0}=B(0,1)$. We denote by $C$ a constant that is independent of the main parameters involved but whose value may differ from line to line. For any index $1<q(x)<\infty$, we denote by $q^{\prime}(x)$ its conjugate index, namely, $q^{\prime}(x)=\frac{q(x)}{q(x)-1}$. For $A \sim D$, we mean that there is a constant $C>0$ such that $C^{-1} D \leq A \leq C D$.

\section{Preliminaries}

In this section, we give the definition of Lebesgue and Herz-Morrey spaces with variable exponent, and give basic properties and useful lemmas.

\subsection{Function spaces with variable exponent}

Let $\Omega$ be a measurable set in $\mathbb{R}^{n}$ with $|\Omega|>0$. We first define variable-exponent Lebesgue spaces.

\section{DEFINITION 2.1}

Let $q(\cdot): \Omega \rightarrow[1, \infty)$ be a measurable function.

(I) The Lebesgue space with variable exponent $L^{q(\cdot)}(\Omega)$ is defined by $L^{q(\cdot)}(\Omega)=\left\{f\right.$ is a measurable function : $F_{q}(f / \eta)<\infty$ for some constant $\left.\eta>0\right\}$, where $F_{q}(f):=\int_{\Omega}|f(x)|^{q(x)} d x$. The Lebesgue space $L^{q(\cdot)}(\Omega)$ is a Banach function space with respect to the norm

$$
\|f\|_{L^{q(\cdot)}(\Omega)}=\inf \left\{\eta>0: F_{q}(f / \eta)=\int_{\Omega}\left(\frac{|f(x)|}{\eta}\right)^{q(x)} d x \leq 1\right\} .
$$

(II) The space $L_{\text {loc }}^{q(\cdot)}(\Omega)$ is defined by $L_{\text {loc }}^{q(\cdot)}(\Omega)=\left\{f\right.$ is measurable $: f \in L^{q(\cdot)}\left(\Omega_{0}\right)$ for all compact subsets $\left.\Omega_{0} \subset \Omega\right\}$.

(III) The weighted Lebesgue space $L_{\omega}^{q(\cdot)}(\Omega)$ is defined as the set of all measurable functions for which

$$
\|f\|_{L_{\omega}^{q(\cdot)}(\Omega)}=\|\omega f\|_{L^{q(\cdot)}(\Omega)}<\infty .
$$

Next we define some classes of variable-exponent functions. Given a function $f \in L_{\text {loc }}^{1}\left(\mathbb{R}^{n}\right)$, the Hardy-Littlewood maximal operator $M$ is defined by 


$$
M f(x)=\sup _{r>0} r^{-n} \int_{B(x, r)}|f(y)| d y,
$$

where $B(x, r)=\left\{y \in \mathbb{R}^{n}:|x-y|<r\right\}$.

\section{DEFINITION 2.2}

Given a measurable function $q(\cdot)$ defined on $\mathbb{R}^{n}$, we write

$$
q_{-}:=\operatorname{essinf}_{x \in \mathbb{R}^{n}} q(x), \quad q_{+}:=\operatorname{ess}_{\sup _{x \in \mathbb{R}^{n}}} q(x) .
$$

(I) $\quad q_{-}^{\prime}=\operatorname{essinf}_{x \in \mathbb{R}^{n}} q^{\prime}(x)=\frac{q_{+}}{q_{+}-1}, q_{+}^{\prime}=\operatorname{essip}_{x \in \mathbb{R}^{n}} q^{\prime}(x)=\frac{q_{-}}{q_{-}-1}$.

(II) Denote by $\mathscr{P}\left(\mathbb{R}^{n}\right)$ the set of all measurable functions $q(\cdot): \mathbb{R}^{n} \rightarrow(1, \infty)$ such that

$$
1<q_{-} \leq q(x) \leq q_{+}<\infty, \quad x \in \mathbb{R}^{n} .
$$

(III) The set $\mathscr{B}\left(\mathbb{R}^{n}\right)$ consists of all measurable functions $q(\cdot) \in \mathscr{P}\left(\mathbb{R}^{n}\right)$ satisfying that the Hardy-Littlewood maximal operator $M$ is bounded on $L^{q(\cdot)}\left(\mathbb{R}^{n}\right)$.

\section{DEFINITION 2.3}

Let $\alpha(\cdot)$ be a real-valued function on $\mathbb{R}^{n}$.

(I) The set $\mathscr{C}_{\operatorname{loc}}^{\log }\left(\mathbb{R}^{n}\right)$ consists of all local log-Hölder continuous functions $\alpha(\cdot)$ satisfying

$$
|\alpha(x)-\alpha(y)| \leq \frac{-C}{\ln (|x-y|)}, \quad|x-y| \leq 1 / 2, x, y \in \mathbb{R}^{n} .
$$

(II) The set $\mathscr{C}_{0}^{\log }\left(\mathbb{R}^{n}\right)$ consists of all log-Hölder continuous functions $\alpha(\cdot)$ satisfying at the origin

$$
|\alpha(x)-\alpha(0)| \leq \frac{C}{\ln \left(e+\frac{1}{|x|}\right)}, \quad x \in \mathbb{R}^{n} .
$$

(III) The set $\mathscr{C}_{\infty}^{\log }\left(\mathbb{R}^{n}\right)$ consists of all log-Hölder continuous functions $\alpha(\cdot)$ satisfying at infinity

$$
\left|\alpha(x)-\alpha_{\infty}\right| \leq \frac{C_{\infty}}{\ln (e+|x|)}, \quad x \in \mathbb{R}^{n},
$$

where $\alpha_{\infty}=\lim _{|x| \rightarrow \infty} \alpha(x)$.

(IV) Denote by $\mathscr{C}^{\log }\left(\mathbb{R}^{n}\right):=\mathscr{C}_{\operatorname{loc}}^{\log }\left(\mathbb{R}^{n}\right) \cap \mathscr{C}_{\infty}^{\log }\left(\mathbb{R}^{n}\right)$ the set of all global logHölder continuous functions $\alpha(\cdot)$.

\section{REMARK 1}

The $\mathscr{C}_{\infty}^{\log }\left(\mathbb{R}^{n}\right)$ condition is equivalent to the uniform continuity condition

$$
|q(x)-q(y)| \leq \frac{C}{\ln (e+|x|)}, \quad|y| \geq|x|, x, y \in \mathbb{R}^{n} .
$$

The $\mathscr{C}_{\infty}^{\log }\left(\mathbb{R}^{n}\right)$ condition was originally defined in this form in [6]. 
Next we define variable-exponent Herz-Morrey spaces $M \dot{K}_{p, q(\cdot)}^{\alpha(\cdot), \lambda}\left(\mathbb{R}^{n}\right)$. Let $B_{k}=$ $\left\{x \in \mathbb{R}^{n}:|x| \leq 2^{k}\right\}, A_{k}=B_{k} \backslash B_{k-1}$, and $\chi_{k}=\chi_{A_{k}}$ for $k \in \mathbb{Z}$.

\section{DEFINITION 2.4}

Suppose that $0 \leq \lambda<\infty, 0<p<\infty, q(\cdot) \in \mathscr{P}\left(\mathbb{R}^{n}\right)$, and $\alpha(\cdot): \mathbb{R}^{n} \rightarrow \mathbb{R}$ with $\alpha(\cdot) \in$ $L^{\infty}\left(\mathbb{R}^{n}\right)$. The variable-exponent Herz-Morrey space $M \dot{K}_{p, q(\cdot)}^{\alpha(\cdot), \lambda}\left(\mathbb{R}^{n}\right)$ is defined by

$$
M \dot{K}_{p, q(\cdot)}^{\alpha(\cdot), \lambda}\left(\mathbb{R}^{n}\right)=\left\{f \in L_{\mathrm{loc}}^{q(\cdot)}\left(\mathbb{R}^{n} \backslash\{0\}\right):\|f\|_{M \dot{K}_{p, q(\cdot)}^{\alpha(\cdot), \lambda}\left(\mathbb{R}^{n}\right)}<\infty\right\}
$$

where

$$
\|f\|_{M \dot{K}_{p, q(\cdot)}^{\alpha(\cdot), \lambda}\left(\mathbb{R}^{n}\right)}=\sup _{k_{0} \in \mathbb{Z}} 2^{-k_{0} \lambda}\left(\sum_{k=-\infty}^{k_{0}}\left\|2^{k \alpha(\cdot)} f \chi_{k}\right\|_{L^{q(\cdot)}\left(\mathbb{R}^{n}\right)}^{p}\right)^{\frac{1}{p}}
$$

Compare the variable Herz-Morrey space $M \dot{K}_{p, q(\cdot)}^{\alpha(\cdot), \lambda}\left(\mathbb{R}^{n}\right)$ with the variable Herz space (see [1]) $\dot{K}_{q(\cdot)}^{\alpha(\cdot), p}\left(\mathbb{R}^{n}\right)$, where

$$
\dot{K}_{q(\cdot)}^{\alpha(\cdot), p}\left(\mathbb{R}^{n}\right)=\left\{f \in L_{\text {loc }}^{q(\cdot)}\left(\mathbb{R}^{n} \backslash\{0\}\right): \sum_{k=-\infty}^{\infty}\left\|2^{k \alpha(\cdot)} f \chi_{k}\right\|_{L^{q(\cdot)}\left(\mathbb{R}^{n}\right)}^{p}<\infty\right\} .
$$

Obviously, $M \dot{K}_{p, q(\cdot)}^{\alpha(\cdot), 0}\left(\mathbb{R}^{n}\right)=\dot{K}_{q(\cdot)}^{\alpha(\cdot), p}\left(\mathbb{R}^{n}\right)$. When $\alpha(\cdot)$ is constant, we have $M \dot{K}_{p, q(\cdot)}^{\alpha(\cdot), \lambda}\left(\mathbb{R}^{n}\right)=M \dot{K}_{p, q(\cdot)}^{\alpha, \lambda}\left(\mathbb{R}^{n}\right)$ (see [30]). If both $\alpha(\cdot)$ and $q(\cdot)$ are constant and $\lambda=0$, then $M \dot{K}_{p, q(\cdot)}^{\alpha(\cdot), \lambda}\left(\mathbb{R}^{n}\right)=\dot{K}_{q}^{\alpha, p}\left(\mathbb{R}^{n}\right)$ are classical Herz spaces.

\subsection{Auxiliary propositions and lemmas}

In this part we state some auxiliary propositions and lemmas which will be needed for proving our main theorems. We only describe the partial results we need.

\section{PROPOSITION 2.1}

Let $q(\cdot) \in \mathscr{P}\left(\mathbb{R}^{n}\right)$.

(I) If $q(\cdot) \in \mathscr{C}^{\log }\left(\mathbb{R}^{n}\right)$, then we have $q(\cdot) \in \mathscr{B}\left(\mathbb{R}^{n}\right)$.

(II) $q(\cdot) \in \mathscr{B}\left(\mathbb{R}^{n}\right)$ if and only if $q^{\prime}(\cdot) \in \mathscr{B}\left(\mathbb{R}^{n}\right)$.

Proposition 2.1(I) is independently due to Cruz-Uribe et al. [6] and Nekvinda [22], respectively. Proposition 2.1(II) belongs to Diening [9] (see also [5, Theorem $1.2])$.

\section{REMARK 2}

Since

$$
\left|q^{\prime}(x)-q^{\prime}(y)\right| \leq \frac{|q(x)-q(y)|}{\left(q_{-}-1\right)^{2}}
$$


it follows at once that if $q(\cdot) \in \mathscr{C}^{\log }\left(\mathbb{R}^{n}\right)$, then so does $q^{\prime}(\cdot)$ - that is, if the condition holds, then $M$ is bounded on $L^{q(\cdot)}\left(\mathbb{R}^{n}\right)$ and $L^{q^{\prime}(\cdot)}\left(\mathbb{R}^{n}\right)$. Furthermore, Diening has proved general results on Musielak-Orlicz spaces.

The order $\beta(x)$ of the fractional Hardy-type operators in Definition 1.1 is not assumed to be continuous. We assume that it is a measurable function on $\mathbb{R}^{n}$ satisfying the following assumptions:

$$
\begin{aligned}
& \beta_{0}:=\operatorname{essinf}_{x \in \mathbb{R}^{n}} \beta(x)>0, \\
& \operatorname{ess} \sup _{x \in \mathbb{R}^{n}} p(x) \beta(x)<n, \\
& \operatorname{ess}_{\sup _{x \in \mathbb{R}^{n}}} p(\infty) \beta(x)<n .
\end{aligned}
$$

In order to prove our main results, we need the Sobolev-type theorem for the space $\mathbb{R}^{n}$ which was proved in [17] for the exponents $p(x)$ not necessarily constant in a neighborhood of infinity, but with some extra power weight fixed to infinity and under the assumption that $p(x)$ takes its minimal value at infinity.

PROPOSITION 2.2

Suppose that $p(\cdot) \in \mathscr{C}^{\log }\left(\mathbb{R}^{n}\right) \cap \mathscr{P}\left(\mathbb{R}^{n}\right)$. Let

$$
1<p(\infty) \leq p(x) \leq p_{+}<\infty,
$$

and let $\beta(x)$ meet condition (2.3). Then the following weighted Sobolev-type estimate is valid for the operator $I_{\beta(\cdot)}$ :

$$
\left\|(1+|x|)^{-\gamma(x)} I_{\beta(\cdot)}(f)\right\|_{L^{q(\cdot)}\left(\mathbb{R}^{n}\right)} \leq C\|f\|_{L^{p(\cdot)}\left(\mathbb{R}^{n}\right)},
$$

where

$$
\frac{1}{q(x)}=\frac{1}{p(x)}-\frac{\beta(x)}{n}
$$

is the Sobolev exponent and

$$
\gamma(x)=C_{\infty} \beta(x)\left(1-\frac{\beta(x)}{n}\right) \leq \frac{n}{4} C_{\infty},
$$

with $C_{\infty}$ being the Dini-Lipschitz constant from (2.2) in which $\alpha(\cdot)$ is replaced by $p(\cdot)$.

\section{REMARK 3}

(i) If $\beta(x)$ satisfies the condition of type (2.2): $\left|\beta(x)-\beta_{\infty}\right| \leq \frac{C_{\infty}}{\ln (e+|x|)}(x \in$ $\left.\mathbb{R}^{n}\right)$, then the weight $(1+|x|)^{-\gamma(x)}$ is equivalent to the weight $(1+|x|)^{-\gamma_{\infty}}$.

(ii) One can also treat operator (1.1) with $\beta(x)$ replaced by $\beta(y)$. In the case of potentials over bounded domains $\Omega$, such potentials differ unessentially if the function $\beta(x)$ satisfies the smoothness logarithmic condition as (2.1), since

$$
C_{1}|x-y|^{n-\beta(y)} \leq|x-y|^{n-\beta(x)} \leq C_{2}|x-y|^{n-\beta(y)}
$$

in this case (see [26, p. 277]). 
(iii) When $p(\cdot) \in \mathscr{P}\left(\mathbb{R}^{n}\right)$, the assumption that $p(\cdot) \in \mathscr{C}^{\log }\left(\mathbb{R}^{n}\right)$ is equivalent to assuming that $1 / p(\cdot) \in \mathscr{C}^{\log }\left(\mathbb{R}^{n}\right)$, since

$$
\begin{aligned}
\left|\frac{p(x)-p(y)}{\left(p_{+}\right)^{2}}\right| & \leq\left|\frac{1}{p(x)}-\frac{1}{p(y)}\right|=\left|\frac{p(x)-p(y)}{p(x) p(y)}\right| \\
& \leq\left|\frac{p(x)-p(y)}{\left(p_{-}\right)^{2}}\right| .
\end{aligned}
$$

Further, $1 / p(\cdot) \in \mathscr{C}^{\log }\left(\mathbb{R}^{n}\right)$ implies that $1 / q(\cdot) \in \mathscr{C}^{\log }\left(\mathbb{R}^{n}\right)$ as well.

The next proposition is the generalization of variable-exponent Herz spaces in [1], and it was used in [21].

\section{PROPOSITION 2.3}

Let $q(\cdot) \in \mathscr{P}\left(\mathbb{R}^{n}\right), p \in(0, \infty)$, and $\lambda \in[0, \infty)$. If $\alpha(\cdot) \in L^{\infty}\left(\mathbb{R}^{n}\right) \cap \mathscr{C}_{0}^{\log }\left(\mathbb{R}^{n}\right) \cap$ $\mathscr{C}_{\infty}^{\log }\left(\mathbb{R}^{n}\right)$, then

$$
\begin{aligned}
\|f\|_{M \dot{K}_{p, q(\cdot)}^{\alpha(\cdot), \lambda}\left(\mathbb{R}^{n}\right)}= & \sup _{k_{0} \in \mathbb{Z}} 2^{-k_{0} \lambda}\left(\sum_{k=-\infty}^{k_{0}}\left\|2^{k \alpha(\cdot)} f \chi_{k}\right\|_{L^{q(\cdot)}\left(\mathbb{R}^{n}\right)}^{p}\right)^{\frac{1}{p}} \\
\approx & \max \left\{\sup _{\substack{k_{0}<0 \\
k_{0} \in \mathbb{Z}}} 2^{-k_{0} \lambda}\left(\sum_{k=-\infty}^{k_{0}} 2^{k \alpha(0) p}\left\|f \chi_{k}\right\|_{L^{q(\cdot)}\left(\mathbb{R}^{n}\right)}^{p}\right)^{\frac{1}{p}}\right. \\
& \sup _{\substack{k_{0} \geq 0 \\
k_{0} \in \mathbb{Z}}}\left(2^{-k_{0} \lambda}\left(\sum_{k=-\infty}^{-1} 2^{k \alpha(0) p}\left\|f \chi_{k}\right\|_{L^{q(\cdot)}\left(\mathbb{R}^{n}\right)}^{p}\right)^{\frac{1}{p}}\right. \\
& \left.\left.+2^{-k_{0} \lambda}\left(\sum_{k=0}^{k_{0}} 2^{k \alpha_{\infty} p}\left\|f \chi_{k}\right\|_{L^{q(\cdot)}\left(\mathbb{R}^{n}\right)}^{p}\right)^{\frac{1}{p}}\right)\right\} .
\end{aligned}
$$

The next lemma is known as the generalized Hölder's inequality on Lebesgue spaces with variable exponent, and the proof can be found in [19].

LEMMA 2.1 (GENERALIZED HÖLDER'S INEQUALITY)

Suppose that $q(\cdot) \in \mathscr{P}\left(\mathbb{R}^{n}\right)$. Then for any $f \in L^{q(\cdot)}\left(\mathbb{R}^{n}\right)$ and any $g \in L^{q^{\prime}(\cdot)}\left(\mathbb{R}^{n}\right)$, we have

$$
\int_{\mathbb{R}^{n}}|f(x) g(x)| d x \leq C_{q}\|f\|_{L^{q(\cdot)\left(\mathbb{R}^{n}\right)}}\|g\|_{L^{q^{\prime}(\cdot)\left(\mathbb{R}^{n}\right)}},
$$

where $C_{q}=1+1 / q_{-}-1 / q_{+}$.

The following lemma can be found in [14].

\section{LEMMA 2.2}

Let $q(\cdot) \in \mathscr{B}\left(\mathbb{R}^{n}\right)$. 
(I) Then there exist positive constants $\delta \in(0,1)$ and $C>0$ such that

$$
\frac{\left\|\chi_{S}\right\|_{L^{q(\cdot)}\left(\mathbb{R}^{n}\right)}}{\left\|\chi_{B}\right\|_{L^{q(\cdot)}\left(\mathbb{R}^{n}\right)}} \leq C\left(\frac{|S|}{|B|}\right)^{\delta}
$$

for all balls $B$ in $\mathbb{R}^{n}$ and all measurable subsets $S \subset B$.

(II) Then there exists a positive constant $C>0$ such that

$$
C^{-1} \leq \frac{1}{|B|}\left\|\chi_{B}\right\|_{L^{q(\cdot)}\left(\mathbb{R}^{n}\right)}\left\|\chi_{B}\right\|_{L^{q^{\prime}(\cdot)}\left(\mathbb{R}^{n}\right)} \leq C
$$

for all balls $B$ in $\mathbb{R}^{n}$.

\section{REMARK 4}

(i) If $q_{1}(\cdot), q_{2}(\cdot) \in \mathscr{C}^{\log }\left(\mathbb{R}^{n}\right) \cap \mathscr{P}\left(\mathbb{R}^{n}\right)$, then we see that $q_{1}^{\prime}(\cdot), q_{2}(\cdot) \in \mathscr{B}\left(\mathbb{R}^{n}\right)$. Hence, we can take positive constants $0<\delta_{1}<1 /\left(q_{1}^{\prime}\right)_{+}, 0<\delta_{2}<1 /\left(q_{2}\right)_{+}$such that

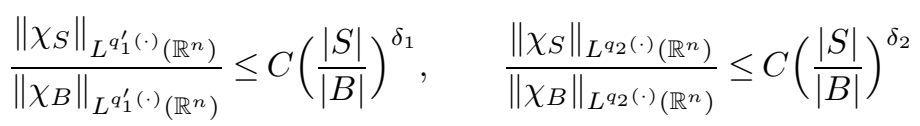

hold for all balls $B$ in $\mathbb{R}^{n}$ and all measurable subsets $S \subset B$ (see [35], [14]).

(ii) On the other hand, Kopaliani [18] has proved the conclusion: if the exponent $q(\cdot) \in \mathscr{P}\left(\mathbb{R}^{n}\right)$ equals a constant outside some large ball, then $q(\cdot) \in \mathscr{B}\left(\mathbb{R}^{n}\right)$ if and only if $q(\cdot)$ satisfies the Muckenhoupt-type condition

$$
\sup _{Q: \text { cube }} \frac{1}{|Q|}\left\|\chi_{Q}\right\|_{L^{q(\cdot)}\left(\mathbb{R}^{n}\right)}\left\|\chi_{Q}\right\|_{L^{q^{(} \cdot(\cdot)\left(\mathbb{R}^{n}\right)}}<\infty .
$$

\section{Main results and their proofs}

Our main result can be stated as follows (for more details see [30]).

\section{THEOREM 3.1}

Suppose that $q_{1}(\cdot) \in \mathscr{C}^{\log }\left(\mathbb{R}^{n}\right) \cap \mathscr{P}\left(\mathbb{R}^{n}\right)$ satisfies condition (2.4), and $\beta(x)$ meets condition (2.3) in which $p(\cdot)$ is replaced by $q_{1}(\cdot)$. Define the variable exponent $q_{2}(\cdot)$ by

$$
\frac{1}{q_{2}(x)}=\frac{1}{q_{1}(x)}-\frac{\beta(x)}{n} .
$$

Let $0<p_{1} \leq p_{2}<\infty$, let $\lambda \geq 0$, and let $\alpha(\cdot) \in L^{\infty}\left(\mathbb{R}^{n}\right)$ be log-Hölder continuous both at the origin and at infinity, with $\alpha(0) \leq \alpha_{\infty}<\lambda+n \delta_{1}$, where $\delta_{1} \in$ $\left(0,1 /\left(q_{1}^{\prime}\right)_{+}\right)$is the constant appearing in (2.6). Then

$$
\left\|(1+|x|)^{-\gamma(x)} \mathscr{H}_{\beta(\cdot)}(f)\right\|_{M \dot{K}_{p_{2}, q_{2}(\cdot)}^{\alpha(\cdot),}\left(\mathbb{R}^{n}\right)} \leq C\|f\|_{M \dot{K}_{p_{1}, q_{1}(\cdot)}^{\alpha(\cdot),}\left(\mathbb{R}^{n}\right)},
$$

where $\gamma(x)$ is defined as in (2.5), and $C_{\infty}$ is the Dini-Lipschitz constant from (2.1) with $q_{1}(\cdot)$ instead of $\alpha(\cdot)$. 
Proof

For any $f \in M \dot{K}_{p_{1}, q_{1}(\cdot)}^{\alpha(\cdot), \lambda}\left(\mathbb{R}^{n}\right)$, if we denote $f_{j}:=f \cdot \chi_{j}=f \cdot \chi_{A_{j}}$ for each $j \in \mathbb{Z}$, then we can write

$$
f(x)=\sum_{j=-\infty}^{\infty} f(x) \cdot \chi_{j}(x)=\sum_{j=-\infty}^{\infty} f_{j}(x) .
$$

By (1.0a) and Lemma 2.1, we have

$$
\begin{aligned}
& \left|\mathscr{H}_{\beta(\cdot)}(f)(x) \cdot \chi_{k}(x)\right| \\
& \quad \leq \frac{1}{|x|^{n-\beta(x)}} \int_{B_{k}}|f(t)| d t \cdot \chi_{k}(x) \\
& \quad \leq C 2^{-k n} \sum_{j=-\infty}^{k}\left\|f_{j}\right\|_{L^{q_{1}(\cdot)}\left(\mathbb{R}^{n}\right)}\left\|\chi_{j}\right\|_{L^{q_{1}^{\prime}(\cdot)}\left(\mathbb{R}^{n}\right)} \cdot|x|^{\beta(x)} \chi_{k}(x) .
\end{aligned}
$$

For Proposition 2.2, we note that

$$
\begin{aligned}
I_{\beta(\cdot)}\left(\chi_{B_{k}}\right)(x) & \geq I_{\beta(\cdot)}\left(\chi_{B_{k}}\right)(x) \cdot \chi_{B_{k}}(x) \\
& =\int_{B_{k}} \frac{1}{|x-y|^{n-\beta(x)}} d y \cdot \chi_{B_{k}}(x) \\
& \geq C|x|^{\beta(x)} \cdot \chi_{B_{k}}(x) \\
& \geq C|x|^{\beta(x)} \cdot \chi_{k}(x) .
\end{aligned}
$$

Using Proposition 2.2, Lemma 2.2, (2.6), (3.1), and (3.2), we have

$$
\begin{aligned}
& \left\|(1+|x|)^{-\gamma(x)} \mathscr{H}_{\beta(\cdot)}(f) \cdot \chi_{k}(\cdot)\right\|_{L^{q_{2}(\cdot)}\left(\mathbb{R}^{n}\right)} \\
& \leq C 2^{-k n} \sum_{j=-\infty}^{k}\left\|f_{j}\right\|_{L^{q_{1}(\cdot)}\left(\mathbb{R}^{n}\right)}\left\|\chi_{j}\right\|_{L^{q_{1}^{\prime}(\cdot)}\left(\mathbb{R}^{n}\right)} \\
& \times\left\|(1+|x|)^{-\gamma(x)} I_{\beta(\cdot)}\left(\chi_{B_{k}}\right)\right\|_{L^{q_{2}(\cdot)\left(\mathbb{R}^{n}\right)}} \\
& \leq C 2^{-k n} \sum_{j=-\infty}^{k}\left\|f_{j}\right\|_{L^{q_{1}(\cdot)}\left(\mathbb{R}^{n}\right)}\left\|\chi_{j}\right\|_{L^{q_{1}^{\prime}(\cdot)}\left(\mathbb{R}^{n}\right)}\left\|\chi_{B_{k}}\right\|_{L^{q_{1}(\cdot)}\left(\mathbb{R}^{n}\right)} \\
& \leq C \sum_{j=-\infty}^{k}\left\|f_{j}\right\|_{L^{q_{1}(\cdot)}\left(\mathbb{R}^{n}\right)} \frac{\left\|\chi_{B_{j}}\right\|_{L^{q_{1}^{(\cdot)}\left(\mathbb{R}^{n}\right)}}}{\left\|\chi_{B_{k}}\right\|_{L^{q_{1}^{\prime}(\cdot)}\left(\mathbb{R}^{n}\right)}} \\
& \leq C \sum_{j=-\infty}^{k} 2^{(j-k) n \delta_{1}}\left\|f_{j}\right\|_{L^{q_{1}(\cdot)}\left(\mathbb{R}^{n}\right)} .
\end{aligned}
$$

Because of $0<p_{1} / p_{2} \leq 1$, applying inequality

$$
\left(\sum_{i=-\infty}^{\infty}\left|a_{i}\right|\right)^{p_{1} / p_{2}} \leq \sum_{i=-\infty}^{\infty}\left|a_{i}\right|^{p_{1} / p_{2}},
$$


and Proposition 2.3, we then have

$$
\begin{aligned}
\left\|(1+|x|)^{-\gamma(x)} \mathscr{H}_{\beta(\cdot)}(f)\right\|_{M \dot{K}_{p_{2}, q_{2}(\cdot)}^{\alpha(\cdot),}\left(\mathbb{R}^{n}\right)}^{p_{1}} & \max _{\substack{\mathfrak{c}_{k_{0}<0} \in \mathbb{Z} \\
k_{0} \in \mathbb{Z}}} 2^{-k_{0} \lambda p_{1}}\left(\sum_{k=-\infty}^{k_{0}} 2^{k \alpha(0) p_{1}}\left\|(1+|x|)^{-\gamma(x)} \mathscr{H}_{\beta(\cdot)}(f) \cdot \chi_{k}\right\|_{L^{q_{2}(\cdot)\left(\mathbb{R}^{n}\right)}}^{p_{1}}\right), \\
& \sup _{\substack{k_{0} \geq 0 \\
k_{0} \in \mathbb{Z}}}\left(2^{-k_{0} \lambda p_{1}}\left(\sum_{k=-\infty}^{-1} 2^{k \alpha(0) p_{1}}\left\|(1+|x|)^{-\gamma(x)} \mathscr{H}_{\beta(\cdot)}(f) \cdot \chi_{k}\right\|_{L^{q_{2}(\cdot)}\left(\mathbb{R}^{n}\right)}^{p_{1}}\right)\right. \\
& \left.\left.+2^{-k_{0} \lambda p_{1}}\left(\sum_{k=0}^{k_{0}} 2^{k \alpha_{\infty} p_{1}}\left\|(1+|x|)^{-\gamma(x)} \mathscr{H}_{\beta(\cdot)}(f) \cdot \chi_{k}\right\|_{L^{q_{2}(\cdot)}\left(\mathbb{R}^{n}\right)}^{p_{1}}\right)\right)\right\} \\
= & \max \left\{E_{1}, E_{2}+E_{3}\right\},
\end{aligned}
$$

where

$$
\begin{aligned}
& E_{1}=\sup _{\substack{k_{0}<0 \\
k_{0} \in \mathbb{Z}}} 2^{-k_{0} \lambda p_{1}}\left(\sum_{k=-\infty}^{k_{0}} 2^{k \alpha(0) p_{1}}\left\|(1+|x|)^{-\gamma(x)} \mathscr{H}_{\beta(\cdot)}(f) \cdot \chi_{k}\right\|_{L^{q_{2}(\cdot)}\left(\mathbb{R}^{n}\right)}^{p_{1}}\right), \\
& E_{2}=\sup _{\substack{k_{0} \geq 0 \\
k_{0} \in \mathbb{Z}}} 2^{-k_{0} \lambda p_{1}}\left(\sum_{k=-\infty}^{-1} 2^{k \alpha(0) p_{1}}\left\|(1+|x|)^{-\gamma(x)} \mathscr{H}_{\beta(\cdot)}(f) \cdot \chi_{k}\right\|_{\left.L^{q_{2}(\cdot)\left(\mathbb{R}^{n}\right)}\right)}^{p_{1}}\right), \\
& E_{3}=\sup _{\substack{k_{0} \geq 0 \\
k_{0} \in \mathbb{Z}}} 2^{-k_{0} \lambda p_{1}}\left(\sum_{k=0}^{k_{0}} 2^{k \alpha_{\infty} p_{1}}\left\|(1+|x|)^{-\gamma(x)} \mathscr{H}_{\beta(\cdot)}(f) \cdot \chi_{k}\right\|_{\left.L^{q_{2}(\cdot)\left(\mathbb{R}^{n}\right)}\right)}^{p_{1}}\right) .
\end{aligned}
$$

To estimate $E_{1}, E_{2}$, and $E_{3}$, we need the following fact. By the condition of $\alpha(\cdot)$ and Proposition 2.3, we have the following cases.

Case $1(j<0)$. We have

$$
\begin{aligned}
\left\|f_{j}\right\|_{L^{q_{1}(\cdot)\left(\mathbb{R}^{n}\right)}} & =2^{-j \alpha(0)}\left(2^{j \alpha(0) p_{1}}\left\|f_{j}\right\|_{L^{q_{1}(\cdot)}\left(\mathbb{R}^{n}\right)}^{p_{1}}\right)^{1 / p_{1}} \\
& \leq 2^{-j \alpha(0)}\left(\sum_{i=-\infty}^{j} 2^{i \alpha(0) p_{1}}\left\|f_{i}\right\|_{L^{q_{1}(\cdot)}\left(\mathbb{R}^{n}\right)}^{p_{1}}\right)^{1 / p_{1}} \\
& \leq 2^{j(\lambda-\alpha(0))}\left(2^{-j \lambda}\left(\sum_{i=-\infty}^{j}\left\|2^{i \alpha(\cdot)} f_{i}\right\|_{L^{q_{1}(\cdot)}\left(\mathbb{R}^{n}\right)}^{p_{1}}\right)^{1 / p_{1}}\right) \\
& \leq C 2^{j(\lambda-\alpha(0))}\|f\|_{M \dot{K}_{p_{1}, q_{1}(\cdot)}^{\alpha(\cdot)}\left(\mathbb{R}^{n}\right)^{\prime}}
\end{aligned}
$$

Case $2(j \geq 0)$. We have

$$
\begin{aligned}
\left\|f_{j}\right\|_{L^{q_{1}(\cdot)}\left(\mathbb{R}^{n}\right)} & =2^{-j \alpha_{\infty}}\left(2^{j \alpha_{\infty} p_{1}}\left\|f_{j}\right\|_{L^{q_{1}(\cdot)}\left(\mathbb{R}^{n}\right)}^{p_{1}}\right)^{1 / p_{1}} \\
& \leq 2^{-j \alpha_{\infty}}\left(\sum_{i=0}^{j} 2^{i \alpha_{\infty} p_{1}}\left\|f_{i}\right\|_{L^{q_{1}(\cdot)}\left(\mathbb{R}^{n}\right)}^{p_{1}}\right)^{1 / p_{1}}
\end{aligned}
$$




$$
\begin{aligned}
& \leq 2^{j\left(\lambda-\alpha_{\infty}\right)}\left(2^{-j \lambda}\left(\sum_{i=-\infty}^{j}\left\|2^{i \alpha(\cdot)} f_{i}\right\|_{L^{q_{1}(\cdot)}\left(\mathbb{R}^{n}\right)}^{p_{1}}\right)^{1 / p_{1}}\right) \\
& \leq C 2^{j\left(\lambda-\alpha_{\infty}\right)}\|f\|_{M \dot{K}_{p_{1}, q_{1}(\cdot)}^{\alpha(\cdot), \lambda}\left(\mathbb{R}^{n}\right)}
\end{aligned}
$$

For $E_{1}$, noting that $j<0$, combining (3.3) and (3.5), and using $\alpha(0) \leq \alpha_{\infty}<$ $\lambda+n \delta_{1}$, we have

$$
\begin{aligned}
E_{1} \leq & C \sup _{\substack{k_{0}<0 \\
k_{0} \in \mathbb{Z}}} 2^{-k_{0} \lambda p_{1}}\left(\sum _ { k = - \infty } ^ { k _ { 0 } } 2 ^ { k \alpha ( 0 ) p _ { 1 } } \left(\sum_{j=-\infty}^{k} 2^{(j-k) n \delta_{1}}\left\|f_{j}\right\|_{\left.\left.L^{q_{1}(\cdot)\left(\mathbb{R}^{n}\right)}\right)^{p_{1}}\right)} \leq\right.\right. \\
\leq & \|f\|_{M \dot{K}_{p_{1}, q_{1}(\cdot)}^{\alpha(\cdot), \lambda}\left(\mathbb{R}^{n}\right)}^{p_{1}} \\
& \times \sup _{\substack{k_{0}<0 \\
k_{0} \in \mathbb{Z}}} 2^{-k_{0} \lambda p_{1}}\left(\sum_{k=-\infty}^{k_{0}} 2^{k \alpha(0) p_{1}}\left(\sum_{j=-\infty}^{k} 2^{(j-k) n \delta_{1}} 2^{j(\lambda-\alpha(0))}\right)^{p_{1}}\right) \\
\leq & C\|f\|_{M \dot{K}_{p_{1}, q_{1}(\cdot)}^{\alpha(\cdot),}\left(\mathbb{R}^{n}\right)}^{p_{1}} \sup _{\substack{k_{0}<0 \\
k_{0} \in \mathbb{Z}}} 2^{-k_{0} \lambda p_{1}}\left(\sum_{k=-\infty}^{k_{0}} 2^{k \lambda p_{1}}\left(\sum_{j=-\infty}^{k} 2^{(j-k)\left(n \delta_{1}+\lambda-\alpha(0)\right)}\right)^{p_{1}}\right) \\
\leq & C\|f\|_{M \dot{K}_{p_{1}, q_{1}(\cdot)}^{\alpha(\cdot), \lambda}\left(\mathbb{R}^{n}\right)}^{\left.p_{1}\right)} \sup _{\substack{k_{0}<0 \\
k_{0} \in \mathbb{Z}}} 2^{-k_{0} \lambda p_{1}}\left(\sum_{k=-\infty}^{k_{0}} 2^{k \lambda p_{1}}\right) \leq C\|f\|_{M \dot{K}_{p_{1}, q_{1}(\cdot)}^{p_{1}(\cdot),}\left(\mathbb{R}^{n}\right)}^{\cdot}
\end{aligned}
$$

We omit the estimate of $E_{2}$ since it is essentially similar to that of $E_{1}$.

Now we only simply estimate $E_{3}$. Noting that $j \geq 0$, combining (3.3) and (3.6), and using $\alpha(0) \leq \alpha_{\infty}<\lambda+n \delta_{1}$, we have

$$
\begin{aligned}
E_{3} & \leq C \sup _{\substack{k_{0} \geq 0 \\
k_{0} \in \mathbb{Z}}} 2^{-k_{0} \lambda p_{1}}\left(\sum_{k=0}^{k_{0}} 2^{k \alpha_{\infty} p_{1}}\left(\sum_{j=-\infty}^{k} 2^{(j-k) n \delta_{1}}\left\|f_{j}\right\|_{L^{q_{1}(\cdot)}\left(\mathbb{R}^{n}\right)}\right)^{p_{1}}\right) \\
& \leq C\|f\|_{M \dot{K}_{p_{1}, q_{1}(\cdot)}^{\alpha(\cdot), \lambda}\left(\mathbb{R}^{n}\right)}^{p_{1}} \sup _{\substack{k_{0} \geq 0 \\
k_{0} \in \mathbb{Z}}} 2^{-k_{0} \lambda p_{1}}\left(\sum_{k=0}^{k_{0}} 2^{k \lambda p_{1}}\left(\sum_{j=-\infty}^{k} 2^{(j-k)\left(n \delta_{1}+\lambda-\alpha_{\infty}\right)}\right)^{p_{1}}\right) \\
& \leq C\|f\|_{M \dot{K}_{p_{1}, q_{1}(\cdot)}^{\alpha(\cdot), \lambda}\left(\mathbb{R}^{n}\right)}^{p_{1} \sup _{k_{0} \geq 0} 2_{0} \in \mathbb{Z}}
\end{aligned}
$$

Combining all the estimates for $E_{i}(i=1,2,3)$ together, we complete the proof of Theorem 3.1.

\section{THEOREM 3.2}

Let $\lambda, p_{1}, p_{2}, q_{1}(\cdot), q_{2}(\cdot), \beta(x), C_{\infty}$ be as in Theorem 3.1. Suppose that $\alpha \in L^{\infty}\left(\mathbb{R}^{n}\right)$ is log-Hölder continuous both at the origin and at infinity, and suppose that $\lambda-$ $n \delta_{2}<\alpha(0) \leq \alpha_{\infty}$, where $\delta_{2} \in\left(0,1 /\left(q_{2}\right)_{+}\right)$is the constant appearing in (2.6). Then

$$
\left\|(1+|x|)^{-\gamma(x)} \mathscr{H}_{\beta(\cdot)}^{*}(f)\right\|_{M \dot{K}_{p_{2}, q_{2}(\cdot)}^{\alpha(\cdot),}\left(\mathbb{R}^{n}\right)} \leq C\|f\|_{M \dot{K}_{p_{1}, q_{1}(\cdot)}^{\alpha(\cdot),}\left(\mathbb{R}^{n}\right)} .
$$


Proof

This is similar to the proof of Theorem 3.1; therefore, we only give a simple proof.

For simplicity, for any $f \in M \dot{K}_{p_{1}, q_{1}(\cdot)}^{\alpha(\cdot), \lambda}\left(\mathbb{R}^{n}\right)$, we write

$$
f(x)=\sum_{j=-\infty}^{\infty} f(x) \cdot \chi_{j}(x)=\sum_{j=-\infty}^{\infty} f_{j}(x) .
$$

By (1.0b) and Lemma 2.1, we have

$$
\begin{aligned}
& \left|(1+|x|)^{-\gamma(x)} \mathscr{H}_{\beta(\cdot)}^{*}(f)(x) \cdot \chi_{k}(x)\right| \\
& \quad \leq C \int_{\mathbb{R}^{n} \backslash B_{k}}|f(t)||x|^{\beta(x)-n} d t \cdot(1+|x|)^{-\gamma(x)} \chi_{k}(x) \\
& \quad \leq C \sum_{j=k+1}^{\infty}\left\|f_{j}\right\|_{L^{q_{1}(\cdot)\left(\mathbb{R}^{n}\right)}}\left\|(1+|x|)^{-\gamma(x)}|\cdot|^{\beta(x)-n} \chi_{j}(\cdot)\right\|_{L^{q_{1}^{\prime}(\cdot)}\left(\mathbb{R}^{n}\right)} \cdot \chi_{k}(x) .
\end{aligned}
$$

Similar to (3.2), we give

$$
I_{\beta(\cdot)}\left(\chi_{B_{j}}\right)(x) \geq I_{\beta(\cdot)}\left(\chi_{B_{j}}\right)(x) \cdot \chi_{B_{j}}(x) \geq C|x|^{\beta(x)} \cdot \chi_{j}(x) .
$$

Applying Proposition 2.2, Lemma 2.2, (2.6), (3.7), and (3.8), we obtain

$$
\begin{aligned}
& \left\|(1+|x|)^{-\gamma(x)} \mathscr{H}_{\beta(\cdot)}^{*}(f) \cdot \chi_{k}(\cdot)\right\|_{L^{q_{2}(\cdot)}\left(\mathbb{R}^{n}\right)} \\
& \leq C \sum_{j=k+1}^{\infty}\left\|f_{j}\right\|_{L^{q_{1}(\cdot)}\left(\mathbb{R}^{n}\right)}\left\|\chi_{k}\right\|_{L^{q_{2}(\cdot)}\left(\mathbb{R}^{n}\right)} \\
& \cdot 2^{-j n}\left\|(1+|x|)^{-\gamma(x)} I_{\beta(\cdot)}\left(\chi_{B_{j}}\right)\right\|_{L^{q_{1}^{\prime}(\cdot)}\left(\mathbb{R}^{n}\right)} \\
& \leq C \sum_{j=k+1}^{\infty}\left\|f_{j}\right\|_{L^{q_{1}(\cdot)}\left(\mathbb{R}^{n}\right)} \frac{\left\|\chi_{B_{k}}\right\|_{L^{q_{2}(\cdot)}\left(\mathbb{R}^{n}\right)}}{\left\|\chi_{B_{j}}\right\|_{L^{q_{2}(\cdot)}\left(\mathbb{R}^{n}\right)}} \\
& \leq C \sum_{j=k+1}^{\infty} 2^{(k-j) n \delta_{2}}\left\|f_{j}\right\|_{L^{q_{1}(\cdot)\left(\mathbb{R}^{n}\right)}} .
\end{aligned}
$$

By (3.4) and Proposition 2.3, we have

$$
\left\|(1+|x|)^{-\gamma(x)} \mathscr{H}_{\beta(\cdot)}^{*}(f)\right\|_{M \dot{K}_{p_{2}, q_{2}(\cdot)}^{\alpha(\cdot),}\left(\mathbb{R}^{n}\right)}^{p_{1}} \leq \max \left\{E_{1}, E_{2}+E_{3}\right\},
$$

where

$$
\begin{aligned}
& E_{1}=\sup _{\substack{k_{0}<0 \\
k_{0} \in \mathbb{Z}}} 2^{-k_{0} \lambda p_{1}}\left(\sum_{k=-\infty}^{k_{0}} 2^{k \alpha(0) p_{1}}\left\|(1+|x|)^{-\gamma(x)} \mathscr{H}_{\beta(\cdot)}^{*}(f) \cdot \chi_{k}\right\|_{L^{q_{2}(\cdot)}\left(\mathbb{R}^{n}\right)}^{p_{1}}\right), \\
& E_{2}=\sup _{\substack{k_{0} \geq 0 \\
k_{0} \in \mathbb{Z}}} 2^{-k_{0} \lambda p_{1}}\left(\sum_{k=-\infty}^{-1} 2^{k \alpha(0) p_{1}}\left\|(1+|x|)^{-\gamma(x)} \mathscr{H}_{\beta(\cdot)}^{*}(f) \cdot \chi_{k}\right\|_{L^{q_{2}(\cdot)\left(\mathbb{R}^{n}\right)}}^{p_{1}}\right), \\
& E_{3}=\sup _{\substack{k_{0} \geq 0 \\
k_{0} \in \mathbb{Z}}} 2^{-k_{0} \lambda p_{1}}\left(\sum_{k=0}^{k_{0}} 2^{k \alpha_{\infty} p_{1}}\left\|(1+|x|)^{-\gamma(x)} \mathscr{H}_{\beta(\cdot)}^{*}(f) \cdot \chi_{k}\right\|_{L^{q_{2}(\cdot)}\left(\mathbb{R}^{n}\right)}^{p_{1}}\right) .
\end{aligned}
$$


For $E_{1}, E_{2}$, and $E_{3}$, combining (3.5), (3.6), and (3.9) and using $\lambda-n \delta_{2}<$ $\alpha(0) \leq \alpha_{\infty}$, we have

$$
E_{i} \leq C\|f\|_{M \dot{K}_{p_{1}, q_{1}(\cdot)}^{\alpha(\cdot),}\left(\mathbb{R}^{n}\right)}^{p_{1}}, \quad i=1,2,3 .
$$

Combining all the estimates for $E_{i}(i=1,2,3)$ together, we complete the proof of Theorem 3.2.

In particular, when $\gamma(x)=0$ and $\alpha(\cdot)$ and $\beta(\cdot)$ are constant exponents, the main results above are proved by Zhang and $\mathrm{Wu}[34]$. Let $\alpha(\cdot)$ be a constant exponent. Then the above results can be found in [30]. When $\lambda=0$, our main results are also valid.

Acknowledgments. The authors cordially thank the anonymous referees who gave valuable suggestions and useful comments which have led to the improvement of this article.

\section{References}

[1] A. Almeida and D. Drihem, Maximal, potential and singular type operators on Herz spaces with variable exponents, J. Math. Anal. Appl. 394 (2012), 781-795. MR 2927498. DOI 10.1016/j.jmaa.2012.04.043.

[2] Y. Chen, S. Levine, and M. Rao, Variable exponent, linear growth functionals in image restoration, SIAM J. Appl. Math. 66 (2006), 1383-1406. MR 2246061. DOI $10.1137 / 050624522$.

[3] M. Christ and L. Grafakos, Best constants for two nonconvolution inequalities, Proc. Amer. Math. Soc. 123 (1995), 1687-1693. MR 1239796.

DOI $10.2307 / 2160978$.

[4] D. Cruz-Uribe, L. Diening, and A. Fiorenza, A new proof of the boundedness of maximal operators on variable Lebesgue spaces, Boll. Unione Mat. Ital. (9) 2 (2009), 151-173. MR 2493649.

[5] D. Cruz-Uribe, A. Fiorenza, J. M. Martell, and C. Pérez, The boundedness of classical operators on variable $L^{p}$ spaces, Ann. Acad. Sci. Fenn. Math. 31 (2006), 239-264. MR 2210118.

[6] D. Cruz-Uribe, A. Fiorenza, and C. J. Neugebauer, The maximal function on variable $L^{p}$ spaces, Ann. Acad. Sci. Fenn. Math. 28 (2003), 223-238.

MR 1976842.

[7] L. Diening, Maximal function on generalized Lebesgue spaces $L^{p(\cdot)}$, Math. Inequal. Appl. 7 (2004), 245-253. MR 2057643. DOI 10.7153/mia-07-27.

[8] , Riesz potential and Sobolev embeddings on generalized Lebesgue spaces and Sobolev spaces $L^{p(\cdot)}$ and $W^{k, p(\cdot)}$, Math. Nachr. 268 (2004), 31-43.

MR 2054530. DOI 10.1002/mana.200310157. 
[9]_ Maximal function on Musielak-Orlicz spaces and generalized Lebesgue spaces, Bull. Sci. Math. 129 (2005), 657-700. MR 2166733.

DOI 10.1016/j.bulsci.2003.10.003.

[10] L. Diening, P. Harjulehto, P. Hästö, Y. Mizuta, and T. Shimomura, Maximal functions in variable exponent spaces: Limiting cases of the exponent, Ann. Acad. Sci. Fenn. Math. 34 (2009), 503-522. MR 2553809.

[11] L. Diening and M. Růžička, Calderón-Zygmund operators on generalized Lebesgue spaces $L^{p(\cdot)}$ and problems related to fluid dynamics, J. Reine Angew. Math. 563 (2003), 197-220. MR 2009242. DOI 10.1515/crll.2003.081.

[12] Z. Fu, Z. Liu, S. Lu, and H. Wang, Characterization for commutators of $n$-dimensional fractional Hardy operators, Sci. China (Ser. A) 50 (2007), 1418-1426. MR 2390459. DOI 10.1007/s11425-007-0094-4.

[13] P. Harjulehto, P. Hästö, V. Latvala, and O. Toivanen, Critical variable exponent functionals in image restoration, Appl. Math. Lett. 26 (2013), 56-60.

MR 2971400. DOI 10.1016/j.aml.2012.03.032.

[14] M. Izuki, Fractional integrals on Herz-Morrey spaces with variable exponent, Hiroshima Math. J. 40 (2010), 343-355. MR 2766665.

[15] M. Izuki and T. Noi, Boundedness of some integral operators and commutators on generalized Herz spaces with variable exponents, OCAMI Preprint Ser. 11 (2011), no. 15 .

[16]_ Duality of Besov, Triebel-Lizorkin and Herz spaces with variable exponents, Rend. Circ. Mat. Palermo (2) 63 (2014), 221-245. MR 3232652. DOI 10.1007/s12215-014-0154-x.

[17] V. Kokilashvili and S. Samko, On Sobolev theorem for Riesz-type potentials in the Lebesgue spaces with variable exponent, Z. Anal. Anwend. 22 (2003), 899-910. MR 2036935. DOI 10.4171/ZAA/1178.

[18] T. S. Kopaliani, Infimal convolution and Muckenhoupt $A_{p(\cdot)}$ condition in variable $L^{p}$ spaces, Arch. Math. (Basel) 89 (2007), 185-192. MR 2341730. DOI 10.1007/s00013-007-2035-4.

[19] O. Kováčik and J. Rákosník, On spaces $L^{p(x)}$ and $W^{k, p(x)}$, Czechoslovak Math. J. 41 (1991), 592-618. MR 1134951.

[20] A. K. Lerner, On some questions related to the maximal operator on variable $L^{p}$ spaces, Trans. Amer. Math. Soc. 362, no. 8 (2010), 4229-4242. MR 2608404. DOI 10.1090/S0002-9947-10-05066-X.

[21] Y. Lu and Y. P. Zhu, Boundedness of multilinear Calderón-Zygmund singular operators on Morrey-Herz spaces with variable exponents, Acta Math. Sin.

(Engl. Ser.) 30 (2014), 1180-1194. MR 3226766.

DOI 10.1007/s10114-014-3410-2.

[22] A. Nekvinda, Hardy-Littlewood maximal operator on $L^{p(x)}\left(\mathbb{R}^{n}\right)$, Math. Inequal. Appl. 7 (2004), 255-265. MR 2057644. DOI 10.7153/mia-07-28.

[23] L. Pick and M. Růžička, An example of a space $L^{p(\cdot)}$ on which the Hardy-Littlewood maximal operator is not bounded, Expo. Math. 19 (2001), 369-371. MR 1876258. DOI 10.1016/S0723-0869(01)80023-2. 
[24] M. Růžička, Electrorheological Fluids: Modeling and Mathematical Theory, Lecture Notes in Math. 1748, Springer, Berlin, 2000. MR 1810360. DOI 10.1007/BFb0104029.

[25] M. Sakharkar and M. Sarde, Image denoising based on variable exponent using adaptive Perona Malik model, Int. J. Eng. Sci. Invent. 3 (2014), 34-37.

[26] S. Samko, Convolution and potential type operators in $L^{p(x)}\left(\mathbb{R}^{n}\right)$, Integral Transforms Spec. Funct. 7 (1998), 261-284. MR 1775832.

DOI 10.1080/10652469808819204.

[27] E E Eratum to "Variable exponent Herz spaces", Mediterr. J. Math. 10 (2013), 2027-2030. MR 3119349. DOI 10.1007/s00009-013-0335-4.

[28] Lariable exponent Herz spaces, Mediterr. J. Math. 10 (2013), 2007-2025. MR 3119348. DOI 10.1007/s00009-013-0285-x.

[29] J. L. Wu, Boundedness of multilinear commutators of fractional Hardy operators, Acta Math. Sci. Ser. A Chin. Ed. 31 (2011), 1055-1062. MR 2866772.

[30] B Boundedness for fractional Hardy-type operator on Herz-Morrey spaces with variable exponent, Bull. Korean Math. Soc. 51 (2014), 423-435.

MR 3194387. DOI 10.4134/BKMS.2014.51.2.423.

[31] J. L. Wu and Q. G. Liu, $\lambda$-central BMO estimates for higher order commutators of Hardy operators, Commun. Math. Res. 30 (2014), 201-206. MR 3242292.

[32] J. L. Wu and J. M. Wang, Boundedness of multilinear commutators of fractional Hardy operators, Appl. Math. J. Chinese Univ. Ser. A 25 (2010), 115-121. MR 2650622.

[33] J. L. Wu and P. Zhang, Boundedness of multilinear Hardy type operators on the product of Herz-Morrey spaces with variable exponent, Appl. Math. J. Chinese Univ. Ser. A 28 (2013), 154-164. MR 3242412.

[34] P. Zhang and J. L. Wu, Boundedness of fractional Hardy type operators on Herz-Morrey spaces with variable exponent, Math. Pract. Theory 43 (2013), 247-254. MR 3114303.

[35] - Boundedness of commutators of fractional Hardy operators on Herz-Morrey spaces with variable exponent, Adv. Math (China) 43 (2014), 581-589. MR 3262223.

Wu: Faculty of Information Technology, Macau University of Science and Technology, Macau, China; Department of Mathematics, Mudanjiang Normal University, Mudanjiang, China; jl-wu@163.com

Zhao: Department of Mathematics, Harbin Institute of Technology, Harbin, China; wenjiaozhao@163.com 